Areg M. Mickaelian*, Gor A. Mikayelyan, Hayk V. Abrahamyan, Gurgen M. Paronyan, and Oleg Yu. Malkov

\title{
Absolute magnitudes of white dwarfs and discovery of new white dwarfs from FBS Blue Stellar Objects by means of Gaia EDR3 data
}

https://doi.org/10.1515/astro-2021-0027

Received Nov 02, 2021; accepted Dec 20, 2021

\begin{abstract}
This article is a presentation of a project which is a statistical study of white dwarfs' properties carried out based on the sample of the First Byurakan Survey Blue Stellar Objects (FBS BSOs) and the Catalogue of White Dwarfs. Using these results, we have searched and will continue to search for new white dwarfs by means of Gaia accurate astrometry. The discovery and study of new White Dwarfs (WDs) is extremely important for understanding the kinematics and dynamics of the local stellar population. They are evolutionary signatures of the Milky Way, as most of stars pass through this evolutionary stage. We have carried out a statistical analysis of the White Dwarfs Catalogue to establish their average physical properties. Based on these data, we have searched for new WDs using Gaia EDR3 accurate astrometry. It provides huge amount of data to confirm WDs and reveal many more such objects. Gaia's astrometric accuracy allows more detailed studies of WDs and many other stars. Using Gaia accurate data in combination with SDSS and DFBS low-dispersion spectra and additional multiwavelength data, we have revealed many new WDs. Many of them are subject for studies on binarity and variability (including a number of subtypes, e.g. ZZ Ceti stars (pulsating WDs), cataclysmic variables, etc.). WDs among FBS blue stellar objects at high galactic latitudes have been searched and revealed. Search for high galactic latitude WDs in DFBS have also been carried out. Using the revealed and calculated characteristics, a statistical study of WD properties is being done.
\end{abstract}

Keywords: blue stellar objects, white dwarfs, cataclysmic variables, accurate astrometry, proper motions, stellar parallaxes, distances

\section{Introduction}

White dwarfs (WDs) are evolutionary signatures of the Milky Way, as most of stars pass through this evolutionary stage. Most of the stars end up their evolution with the stage of WDs, so it is considered that such type of objects should be widely spread in the Galaxy. Moreover, all new discoveries prove that the number of WDs (and many other dwarf stars) is much higher than the previously adopted numbers; in fact, with the discovery of more stars in the Galaxy, especially the number (and hence, the fraction) of new WDs

Corresponding Author: Areg M. Mickaelian: NAS RA V. Ambartsumian Byurakan Astrophysical Observatory (BAO), Armenia; Email: aregmick@yahoo.com

Gor A. Mikayelyan, Hayk V. Abrahamyan, Gurgen M. Paronyan: NAS RA V. Ambartsumian Byurakan Astrophysical Observatory (BAO), Armenia

Oleg Yu. Malkov: Institute of Astronomy, Moscow, Russia

כ Open Access. @ 2021 A. M. Mickaelian et al., published by De Gruyter. 4.0 License become larger. The McCook and Sion (2016) catalogue of WDs gives the list of spectroscopically identified objects. For each degenerate star, the following data entries with references are provided: catalog coordinate designation or WD number, in order of right ascension; R.A. and DEC for epoch 1950.0; the spectral type based upon the new system; a catalog symbol denoting binary membership; a list of most names known to exist for a given star; proper motion and position angle; broadband UBV photometry, V, B-V, U-B multichannel spectrophotometry, v(MC), g-r; Strömgren narrowband photometry, y, b-y, u-b; an absolute visual magnitude based upon the best available colormagnitude calibration or trigonometric parallax; the observed radial velocity uncorrected for gravitational redshift or solar motion; and the trigonometric parallax with mean error when available. It is worth mentioning that there are several newer catalogues of WDs, particularly based on the Sloan Digital Sky Survey (SDSS; Ahumada et al. (2020)), namely Kepler et al. (2015) and Kepler et al. (2019). In the 
latter one, there are 20,088 spectroscopically confirmed WDs. SDSS has been accomplished over 14,555 $\mathrm{deg}^{2}$ area and does not represent the entire sky, though its data are extremely accurate and useful. A catalogue of white dwarfs in Gaia EDR3 was published as well (Gentile Fusillo et al. 2021), also not used in the present paper. According to the authors, a sample of $\sim 359,000$ high-confidence white dwarf candidates have been selected.

The discovery and study of new WDs is extremely important for understanding the kinematics and dynamics of the local stellar population. Especially many new possibilities are connected with Gaia space observatory (Prusti et al. (2016), and Brown et al. (2021) for EDR3). Gaia is an astrometric project of ESA, launched in 2013 and expected to operate until 2022 . It will be used for measuring the positions, distances and motions of stars with unprecedented precision, which will be especially useful for discovery and study of many thousands of WDs. The spectrophotometric measurements will provide the detailed physical properties of all stars observed, characterizing their luminosity, effective temperature, gravity and elemental composition.

The First Byurakan Survey (FBS) $2^{\text {nd }}$ Part was devoted to search and studies of Blue Stellar Objects (BSOs; Mickaelian (2008)) and Late-type Stars. Eleven lists of 1103 BSOs were published in Astrophysics in 1990-1996, found in FBS low-dispersion spectroscopic plates. The selection was carried out in the region with $+33^{\circ}<\delta<+45^{\circ}$ and $\delta>+61^{\circ}$ with a surface of $\sim 4000 \mathrm{deg}^{2}$. As a result, the catalogue of the FBS BSOs was compiled. Its preliminary version has been available at CDS since 1999. We revised and updated the FBS BSOs catalogue with the new data from recently published optical and multiwavelength catalogues to give access to all available data and make further comparative studies of the properties of these objects possible. We have made cross-correlations of the FBS BSOs catalogue with the MAPS, USNO-B1.0, SDSS, and 2MASS, as well as ROSAT, IRAS, NVSS, and FIRST catalogs, added updated SIMBAD and NED data for the objects, and provided accurate DSS1 and DSS2 positions and revised photometry. We also checked the objects for proper motion and variability (Mickaelian and Sinamyan 2010). A refined classification for the low-dispersion spectra in the Digitized First Byurakan Survey (DFBS) was carried out. The revised and updated catalogue of FBS blue stellar objects contains 1101 objects. The FBS blue stellar objects catalogue can be used to study a complete sample of white dwarfs, hot subdwarfs, HBB stars, cataclysmic variables, bright AGN, and to investigate individual interesting objects.

\section{Statistical analysis of the Catalogue of White Dwarfs}

We have analyzed The Catalogue of Spectroscopically Identified White Dwarfs (Version April 2014) by McCook and Sion (2016) (hereafter MS-2014) to summarize the statistical properties of WDs. Though many WDs have been later discovered in SDSS, however, MS-2014 is an all-sky catalogue and gives brighter objects for studies of the Galactic structure and kinematics in the Solar neighborhood.

The total number of WDs in MS-2014 this version is 14,294. Among them there are:

- 368 WDs having parallaxes, in the range 0.0001-0.575, the average is 0.046 ;

- 10,871 WDs having total proper motion (PM) in the range of 0.0010-4.0800 arcsec/year;

- only 345 have adopted apparent velocities (Adp-V) in the range of $0.058-97.190 \mathrm{~km} / \mathrm{s}$, among them 328 (95.1\%) have velocities less than $75 \mathrm{~km} / \mathrm{s}$;

- 1937 WDs have absolute magnitudes in the range of $-0.11 \ldots$. 18.10; the distribution by absolute magnitudes shows that we can consider as the limit for WD $7.5^{m}$ (and even $9.5^{m}$, as brighter WDs are extremely rare);

- 1919 WDs (out of 1937, 99.1\%) are fainter than $7.5^{m}$ or even $9.5^{m}$ (1792 out of 1937, 92.5

As MS-2016 is a collection of all available data at the moment, there is no homogeneity. However, it is useful to derive statistical properties of WDs for further use. Here the authors use the distribution over adopted apparent velocities to derive fractions of objects having the given velocity. In Figure 1 we give the distribution of absolute magnitudes (left panel) and adopted apparent velocities (right panel) for McCook and Sion Catalogue WDs allowing estimate the limit of $M_{\text {abs }}$ for WDs (two breaks at $7.5^{m}$ and $9.5^{m}$ ) and the limit of the apparent velocity (the break at 75 $\mathrm{km} / \mathrm{s}$ ). Adopting these values, we could distinguish WDs from the other stars.

Let us note that the WD sample is incomplete after $11.5^{\mathrm{m}}$ (the distribution graph goes down) due to the difficulty of discovery of very faint objects, especially significant for WDs.

In Figure 2 we give the distribution of the parallaxes vs. proper motions for McCook and Sion WDs. The dependence is obvious and one can roughly estimate the parallax based on the measured proper motion, hence have understanding on the distance. The absolute magnitude can be derived accordingly. This was used as our preliminary approach to obtain distances using proper motions. And only lower 

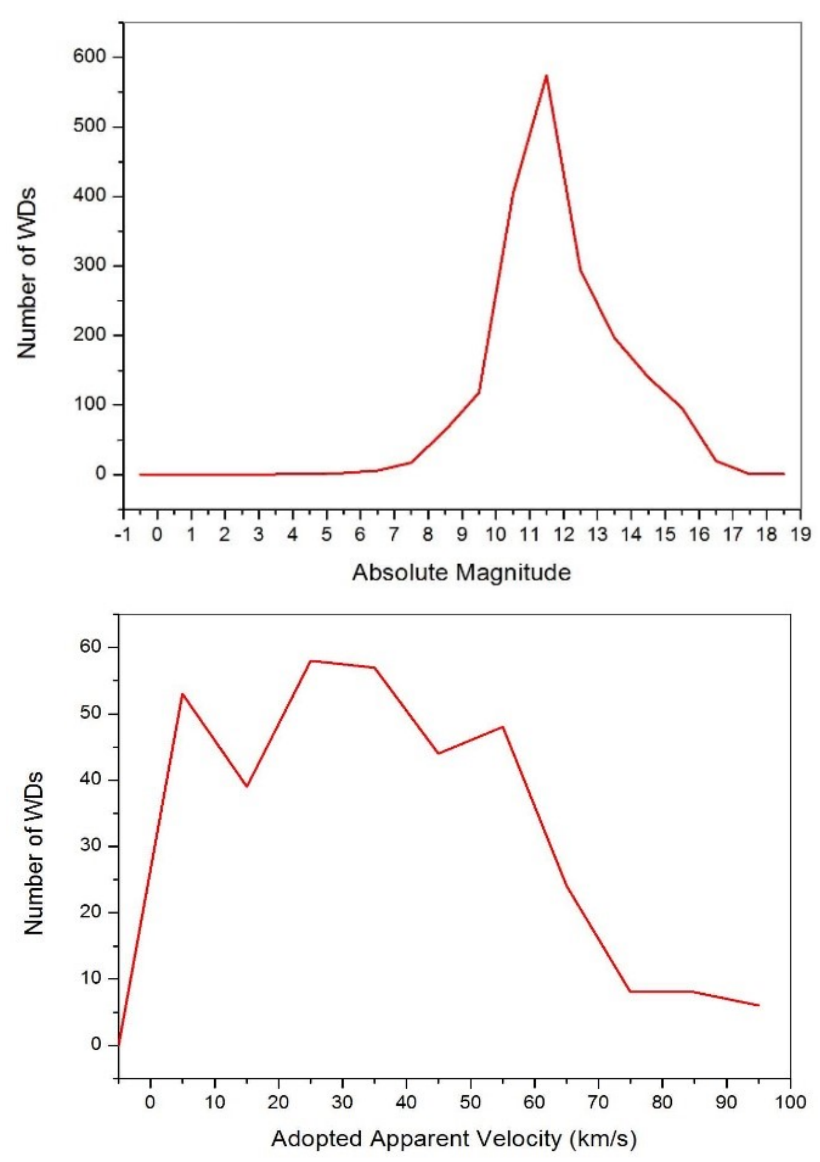

Figure 1. Distribution of absolute magnitudes and adopted apparent velocities for McCook and Sion Catalogue WDs

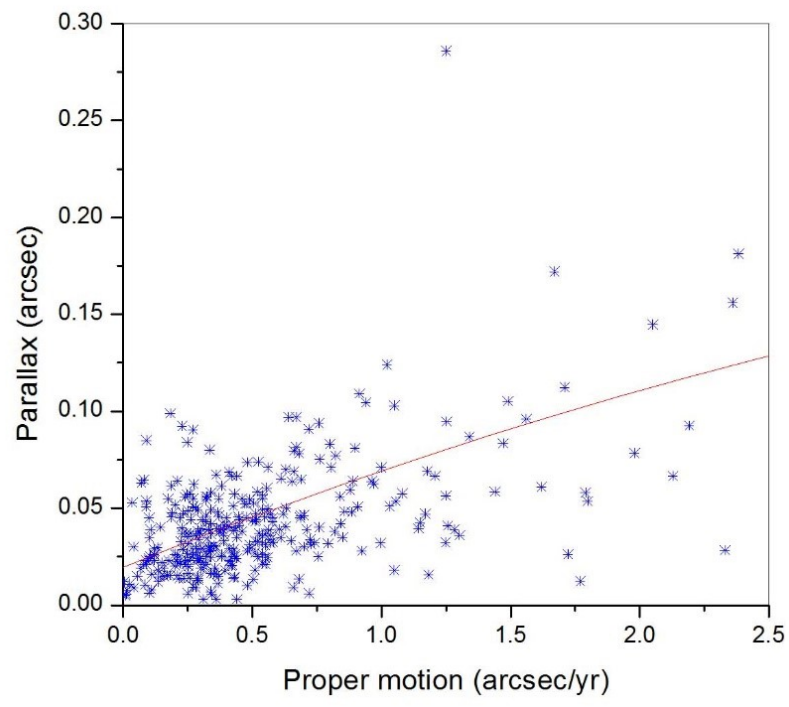

Figure 2. Distribution of parallaxes vs. proper motions for McCook and Sion Catalogue WDs

limits (hence, upper limits of luminosities) were used to estimate if this was WD or not.
As a result, we find the following characteristics typical of white dwarfs absolute magnitudes $M_{\text {abs }}: 7.5^{m}$ as a strict criterion for all objects and $9.5^{m}$ as a criterion for vast the majority of objects. Concerning the colors, MS-2016 contains relatively later type WDs as well, however, according to our FBS blue stellar objects list (containing mostly DO, DB and DA WDs), average B-R is 0.044 , and the range is from -1.30 to +2.34 , however $93.3 \%$ of objects have colors between -1.3 and +1.0 . Some objects are outliers, which may mean both that 1) the limits are not very strict and some small number may still have broader range of these characteristics or 2) these are exotic objects. We used the derived characteristics to look for new WDs in other databases.

\section{Gaia EDR3 data and SDSS photometry and spectra}

We have carried out cross-correlations of WDs with Gaia EDR3 (Brown et al. 2021) for parallaxes, PM, distances and Teff, as well as cross-correlations with SDSS DR16 (Ahumada et al. 2020) for photometry (magnitudes and colors) and spectra. The search radius was 5 arcsec in both cases. For distances, Gaia EDR3 catalogue (Bailer-Jones et al. 2021) was used giving data on 1.47 billion stars. Here are the numbers of matches:

- Gaia EDR3: 4338 matches,

- Gaia distances: 4233 matches,

- SDSS DR16: 4468 matches.

Here we should note that due to their large proper motions, it is rather difficult to associate many of the objects. As Gaia observations are shifted by the epoch from 2000.0 more that those of the SDSS, Gaia associations turn to be less.

Thus, for the vast majority (98\%) of the WDs present in Gaia EDR3 we have distances. Moreover, we could derive absolute magnitudes and luminosities for the found objects and refine their data. In fact, both data (Gaia distances and derived distances from absolute magnitudes) complement each other.

SDSS data are rather useful for multicolor photometry, as well as for spectroscopy for many of the objects (to derive their spatial velocities using also proper motions and distances). SDSS itself also gives data on proper motions and this is an additional option to complement Gaia data, though with lower accuracy.

In Figure 3, we give the SDSS color-color diagram for McCook and Sion Catalogue 4468 WDs, where most of the 
objects are concentrated in the lower left region (relatively bluer objects, typical of WDs).

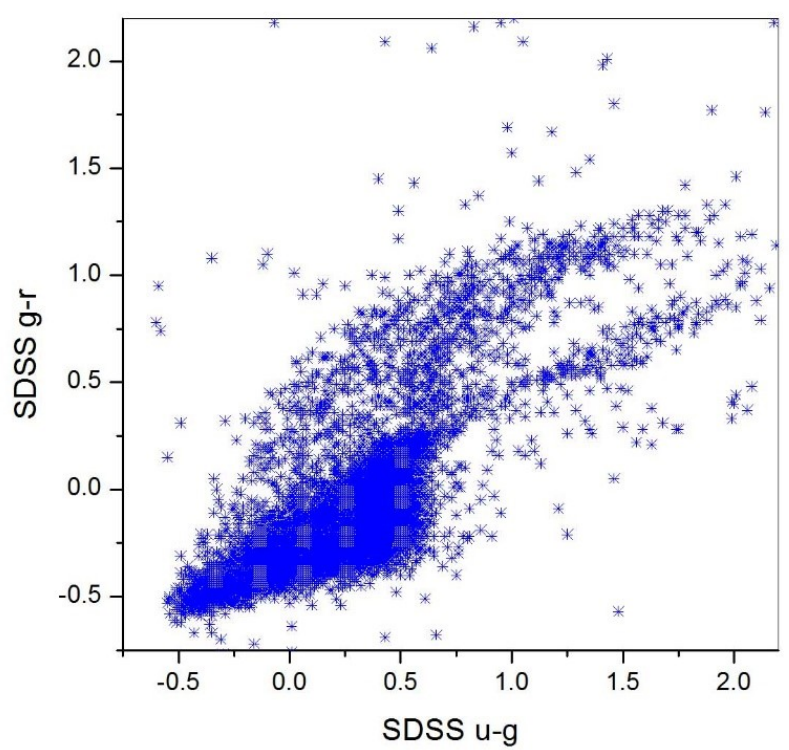

Figure 3. SDSS color-color diagram for McCook and Sion Catalogue 4468 WDs

\section{FBS Blue Stellar Objects, their proper motions and white dwarfs among them}

The First Byurakan Survey (FBS) Blue Stellar Objects (BSOs) are the result of the search of stellar objects with the same Markarian criteria (as used for selection of UV-excess galaxies) in the FBS plates. Markarian used an objective prism survey (FBS) and examined with a lens all low-dispersion spectra in the photographic plates. There were 1874 plates and each plate contained some 15,000-20,000 spectra. Among them, some had excess in the UV part $(\lambda=3400$ 3900 AA). These galaxies with higher resolution spectroscopic observations appeared to be rather interesting objects: Seyferts, LINERs, Starbursts, etc. FBS $2^{\text {nd }}$ Part was devoted to search and revelation of stellar objects applying Markarian criteria to star-like spectra. Many hot subdwarfs, white dwarfs, Horizontal Branch B (HBB) stars, cataclysmic variables, Planetary Nebulae Nuclei (PNN), as well as QSOs and Seyferts (with compact images) were revealed. Later on, the Digitized FBS (DFBS) database was used both to refine the criteria of selection and for search for many new (fainter) objects. 185 WDs have been revealed (Mickaelian
2003, 2005; Sinamyan and Mickaelian 2011), including by using the astrometry first from APM, USNO, MAPS and GSC databases and then using the accurate astrometry from Gaia. MAPS color-magnitude diagram for FBS BSOs is given in Figure 4. One may suppose that WDs are distinguishable on the lower left part of the diagram, as well as hot subdwarfs, somewhat upper from this locus.

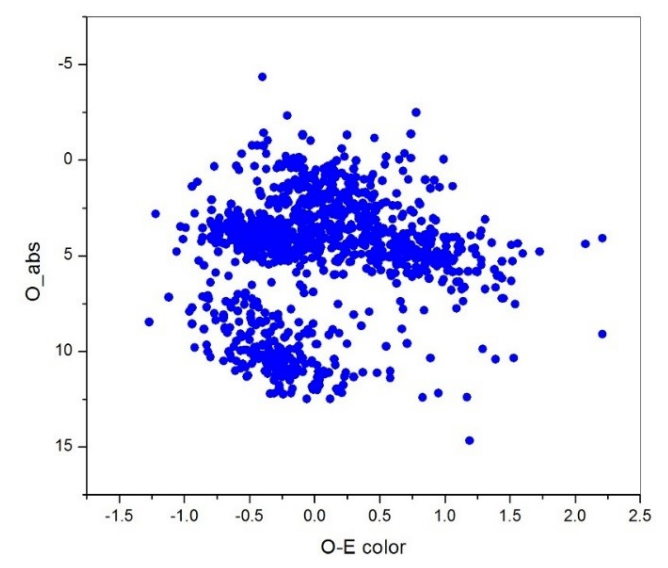

Figure 4. MAPS color-magnitude diagram for FBS BSOs. WDs are easily distinguishable on the lower left part of the diagram, as well as hot subdwarfs, somewhat upper from this locus

Two examples of WDs low-dispersion spectra (both 2D and 1D extraction) are given in Figure 5. The extraction is done using the dedicated software bSpec ("Byurakan spectra"). They are presented at the Digitized First Byurakan Survey (DFBS) webpage at https://www.aras.am/ /Dfbs/SampleSpectra/wd3.htm (DFBS 2007), together with other types that obviously differ from these spectra.

Cross-correlation of the Revised and updated First Byurakan Survey (FBS) 1103 objects was also done with the same three databases (the search radius was 5 arcsec):

- Gaia EDR3: 1032 matches,

- Gaia distances: 1028 matches,

- SDSS DR16: 631 matches.

As for MS-2014 objects, FBS BSOs also have large proper motions and many objects could have not been found due to this effect, as many objects are shifted from their search positions. In case of the SDSS, some Northern regions are absent compared to the FBS (Galactic latitude between $15^{\circ}$ and $\left.30^{\circ}\right)$, as well as some Southern declinations $\left(-15^{\circ}<\right.$ $\left.\delta<0^{\circ}\right)$. That is why only half of the objects are found in SDSS.

At present search for WDs in DFBS has been conducted. Thousands of new objects are expected. Gaia is especially useful and efficient to reveal WDs (and hot subdwarfs) 

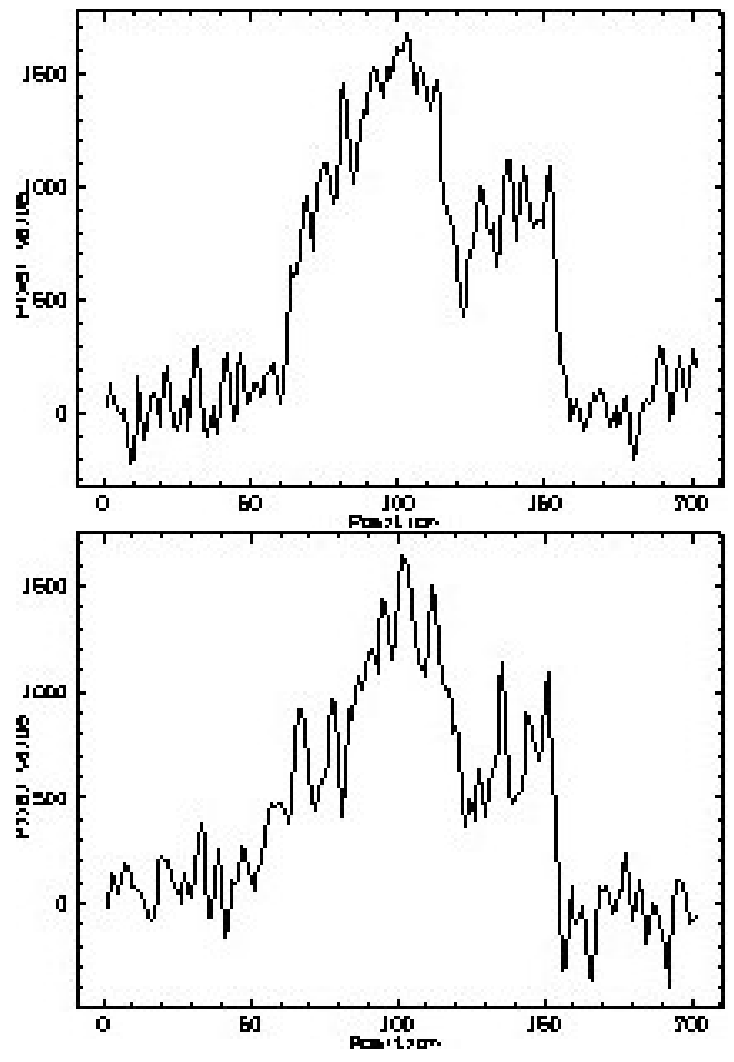

Figure 5. DFBS low-dispersion spectra for two genuine WDs (PG 0109+111 and PG 1449+168) showing the shape of their spectra. Both 2D and 1D extractions are given

among the blue stellar objects, as very few of these objects are giants/supergiants. Note, that there is a small number of Horizontal Branch Blue (HBB) stars as well.

\section{Results and summary}

We have carries out a statistical study of the samples of McCook and Sion WD catalogue and FBS blue stellar objects using Gaia and SDSS data; accurate astrometry and multiband photometry and spectroscopy, respectively.

One can summarize our results as follows:

- we have carried out statistical analysis of McCook and Sion WD Catalogue: we have established the range and average numbers for absolute magnitudes, colors, proper motions, $\mathrm{T}_{\text {eff }}$, etc.; the limits of adopted tangential velocities for calculation of the absent proper motions were derived. We have estimated the lower (brighter) limit for absolute magnitudes and proved that such blue stars can only be WDs;
- we have also carried out a statistical study of the FBS blue stellar objects sample to reveal new WDs among these stars. Identifying all WDs among the FBS blue stellar objects, 185 in total have been revealed (based on our previous work and confirmed by Gaia data);

- we have analyzed the possibility and established parameters to reveal thousands of new WD candidates from Gaia EDR3 accurate astrometry.

Further studies include:

- revealing thousands of new spectroscopically identified WDs at high Galactic latitudes from SDSS, based on their proper motions and spectroscopy;

- revealing thousands of new high Galactic latitude WD candidates in the DFBS using both DFBS and Gaia low-dispersion spectra, when available.

Acknowledgment: We thank the anonymous referee for valuable comments and suggestions. The authors (AMM, GAM, HVA and GMP) are grateful to the ANSEF foundation for ANSEF grant PS-astroex-2512 "Discovery and study of new white dwarfs using Gaia accurate astrometry" (20212022). O. M. acknowledges support from the Russian Foundation for Basic Researches (project 20-52-53009). SDSS DR16 and Gaia EDR3 catalogue teams are also acknowledged.

Author contributions: All authors have accepted responsibility for the entire content of this manuscript and approved its submission.

Conflict of interest: The authors state no conflict of interest.

\section{References}

Ahumada R, Prieto CA, Almeida A, Anders F, Anderson SF, Andrews BH, et al. 2020. The 16th Data Release of the Sloan Digital Sky Surveys: First Release from the APOGEE-2 Southern Survey and Full Release of eBOSS Spectra. ApJ Suppl Ser. 249(1):3.

Bailer-Jones CAL, Rybizki J, Fouesneau M, Demleitner M, Andrae R. 2021. Distances to 1.47 billion stars in Gaia EDR3. VizieR Online Data Catalog: I/352.

Brown AGA, Vallenari A, Prusti T, de Brujine JHJ, Babusiaux C, BailerJones CAL. 2021. Gaia Data Release 2. Summary of the contents and survey properties. A\&A. 650(C3).

Gentile Fusillo NP, Tremblay PE, Cukanovaite E, Vorontseva A, Lallement R, Hollands M, et al. 2021. A catalogue of white dwarfs in Gaia EDR3. Mon Not R Astron Soc. 508(3):3877-3896.

Kepler SO, Pelisoli I, Koester D, Ourique G, Kleinman SJ, Romero $A D$, et al. 2015. New white dwarf stars in the Sloan Digital Sky 
Survey Data Release 10. Mon Not R Astron Soc. 446(4):40784087.

Kepler SO, Pelisoli I, Koester D, Reindl N, Geier S, Romero AD, et al. 2019. White dwarf and subdwarf stars in the Sloan Digital Sky Survey Data Release 14. Mon Not R Astron Soc. 2019;486(2):2169-2183.

McCook GP, Sion EM. 2016. Spectroscopically identified white dwarfs. VizieR Online Data Catalog.

Mickaelian AM. 2003. New white dwarfs and cataclysmic variables from the FBS. IAU Joint Discussion. 25(E5).

Mickaelian AM. 2005. White dwarfs and cataclysmic variables in the FBS. Astrophysics and Space Science Library. In: Sion EM, Vennes S, Shipman HL, Editors. White dwarfs: cosmological and galactic probes. Dordrecht: Springer. p. 61-72.
Mickaelian, A. M. 2008. Revised and updated catalogue of the First Byurakan Survey blue stellar objects. Astron J, 136:946.

Mickaelian AM, Sinamyan PK. 2011. Proper motions of FBS blue stellar objects. VizieR Online Data Catalog.

Prusti T, de Bruijne JHJ, Brown AGA, Vellnari A, Babusiaux C, BailerJones CAL, et al. 2016. The Gaia mission. A\&A 595(A1).

Sinamyan PK, Mickaelian AM. 2011. A subsample of white dwarfs among the FBS blue stellar objects. Astrophysics. 54:403-412. 